\title{
Proposition d'activité pédagogique pour mieux appréhender les perspectives éthiques anthropocentrique, biocentrique
}

Carine Rousseau

\section{OpenEdition}

\section{Journals}

Édition électronique

URL : http://journals.openedition.org/ere/539

DOI : 10.4000/ere.539

ISSN : 2561-2271

Éditeur

Centr'ERE

Référence électronique

Carine Rousseau, « Proposition d'activité pédagogique pour mieux appréhender les perspectives

éthiques anthropocentrique, biocentrique », Éducation relative à l'environnement [En ligne], Volume 12 I

2015, mis en ligne le 20 mai 2015, consulté le 10 décembre 2020. URL : http://

journals.openedition.org/ere/539; DOI : https://doi.org/10.4000/ere.539 


\title{
Proposition d'activité pédagogique pour mieux appréhender les perspectives éthiques anthropocentrique, biocentrique
}

\author{
Carine Rousseau
}

Devant une problématique socio-environnementale complexe, la collaboration entre différents acteurs de différents domaines est nécessaire. Notre postulat est que celle-ci serait facilitée si les participants étaient capables de comprendre la vision du monde de chacun des acteurs et de mieux saisir leurs différentes identités comme agents environnementaux. Ceci pourrait les aider à mieux cerner comment intervenir ensemble pour poser des gestes concrets. Dans cet article, nous proposons une activité pédagogique à mener avec des enfants du primaire qui pourrait leur permettre de mieux appréhender la vision du monde qui sous-tend leurs propres choix en matière d'environnement, mais aussi celles de leurs pairs. Cette vision sera abordée sous l'angle de l'éthique sur laquelle elle se fonde. Après avoir expliqué le contexte de cette recherche, nous décrirons brièvement les trois perspectives en éthiques de l'environnement que nous avons contrastées, pour ensuite expliquer la construction des textes qui servent de support à l'activité que nous présenterons.

\section{Contexte}

Dans le cadre de notre mémoire de maîtrise en éducation relative à l'environnement (Rousseau, 2011), nous avons conçu plusieurs questions et activités portant sur la dimension éthique du rapport à l'environnement. Celles-ci furent élaborées à partir de notre cadre théorique et de l'analyse-critique d'un questionnaire, le NEP Scale for Children de Manoli, Johnson et Dunlap, 2007, inspirée de à l'étude qu'en avait faite Lundmark (2007). 
Dans ce court texte, nous ne pourrons pas présenter la totalité des questions et des activités proposées. Nous choisissons plutôt d'expliquer plus en détail l'activité synthèse : "Les trois récits ». Il s'agit de trois textes courts décrivant un même acte en faveur de l'environnement (planter un arbre), mais découlant de motivations différentes (issues de trois différentes perspectives éthiques). Cet exercice permettrait non seulement aux participants de s'identifier à une perspective, mais aussi d'analyser les visions des autres, tout en mettant de l'avant que, malgré leurs différences, ils peuvent choisir d'accomplir le même acte.

\section{Brève description de trois perspectives en éthique de l'environnement}

4 Selon Sauvé et Villemagne (2003), chaque éthique environnementale témoignerait d'une certaine vision de l'environnement et de notre rapport à celui-ci. Ces auteures caractérisent entre autres trois perspectives: l'anthropocentrisme (centré sur l'être humain), le biocentrisme (centré sur la vie) et l'écocentrisme (centré sur les systèmes de support à la vie). Selon ces auteures, le porteur d'une éthique anthropocentrique chercherait à préserver l'environnement pour protéger des intérêts humains (par exemple, conserver des ressources naturelles ou un lieu récréatif ou esthétiquement beau) et/ou pour assurer la survie de l'espèce humaine (prise en compte des générations futures). Selon cette perspective, les êtres humains ne considèrent les animaux et les végétaux que suivant les bénéfices qu'ils peuvent leur apporter. Au contraire, la perspective biocentriste leur accorde une valeur intrinsèque du fait qu'ils sont des êtres vivants. Nous aurions alors, selon cette perspective, des devoirs moraux de protection envers eux. L'écocentrisme, selon Afeissa (2007), va dans le même sens, mais ajoute également l'importance des éléments abiotiques et de systèmes plus vastes: la perspective est écosystémique (incluant des végétaux, des animaux, mais aussi des habitats, des montages, etc.). Cette décentration des humains ne se ferait pas au détriment de ces derniers, car selon Callicott (1999), nous sommes d'abord responsables des êtres et des systèmes qui nous sont proches. Cette perspective place, comme le biocentrisme, l'être humain parmi les animaux, mais elle fait référence à une vision plus large de l'environnement, car elle y inclut également les réalités et les réalisations humaines. Sont ainsi mises de l'avant les relations et dépendances réciproques entre les systèmes naturels et sociaux. Nous parlerons alors, pour reprendre les termes de Goffin (1998), d'éco-socio-système.

Dans le cadre théorique de notre maitrise, nous comparons ces trois perspectives en ce qui les différencie et les rassemble. De ce travail, nous avons mis en évidence quatre thèmes ${ }^{1}$ de comparaison : les conceptions de la nature et de l'environnement, les droits des êtres vivants, la place des humains dans ou par rapport à la nature et ce qui nous préoccupe quand nous réfléchissons à l'avenir de l'environnement (Êtres humains uniquement? Animaux ? Végétaux ?) (Rousseau, 2011 et 2013).

\section{Construction des textes}

6 Notre but était donc de construire trois récits portant sur le même acte environnemental (planter un arbre), mais chacun reflétant l'une des trois perspectives 
différentes. Il s'agit d'inciter les enfants à mieux cerner leur propre vision et de leur offrir un support pour échanger leurs points de vue avec ceux de leurs pairs.

Pour cela, nous avons d'abord élaboré un tableau (Tableau 1) faisant ressortir les éléments importants des trois perspectives selon les thèmes choisis. Puis nous avons utilisé ces éléments pour construire chacun des récits. Ainsi le récit d'Arthur reflète une vision anthropocentrique, celui de Bastien une vision biocentrique et celui d'Éric une vision écocentrique. Afin de faciliter la lecture et le repérage des différents éléments dans les textes, nous avons indiqué pour chaque phrase des récits à quelle perspective (anthropocentrique, biocentrique ou écocentrique) celle-ci référait. Ainsi les phrases indiquées par un $\mathrm{C}$ font référence aux conceptions de la nature et de l'environnement, celles par un $\mathbf{D}$ aux droits de la nature, celles par un $\mathbf{H}$ à la place des humains dans ou par rapport à la nature et celles par un $\mathbf{A}$ à l'avenir de l'environnement. Les numéros suivant les lettres indiquent plus précisément à quel élément chaque phrase renvoie dans les différentes perspectives. Par exemple, l'élément $\mathrm{C} 2$ du récit d'Arthur a été élaboré à partir de l'élément $\mathrm{C} 2$ du tableau 1.

Tableau 1 : Éléments anthropocentrique, biocentrique et écocentrique dans les trois récits suivants les thèmes choisis

\begin{tabular}{|c|c|c|c|}
\hline & $\begin{array}{l}\text { Anthropocentrisme } \\
\text { (récit d'Arthur) }\end{array}$ & $\begin{array}{c}\text { Biocentrisme } \\
\text { (récit de Bastien) }\end{array}$ & $\begin{array}{l}\text { Écocentrisme } \\
\text { (récit d'Êric) }\end{array}$ \\
\hline 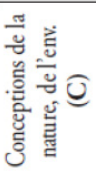 & $\begin{array}{l}\text { Nature davantage vue comme une } \\
\text { ressource }(\mathbf{C} 1) \text { ou comme un lieu } \\
\text { de détente, de distractions ou } \\
\text { esthétiquement beau }(\mathbf{C} 2) \text {. }\end{array}$ & $\begin{array}{l}\text { Nature dont les humains font } \\
\text { partie, importance mise sur la vie, } \\
\text { sur les êtres vivants (C3). }\end{array}$ & $\begin{array}{l}\text { Éco-socio-système qui inclut des } \\
\text { éléments construits par les humains } \\
\text { (C4). Présence d'une certaine } \\
\text { dynamique entre les dimensions } \\
\text { sociale et culturelle (C5). }\end{array}$ \\
\hline 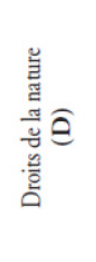 & $\begin{array}{l}\text { Distinction entre les humains } \\
\text { (supérieurs) et le reste des êtres } \\
\text { vivants (D1). } \\
\text { Préserver les ressources naturelles } \\
\text { pour nous-mêmes et les générations } \\
\text { futures (D2). } \\
\text { Responsabilité envers les humains et } \\
\text { les générations futures (D2). }\end{array}$ & $\begin{array}{l}\text { Les êtres vivants ont une valeur } \\
\text { intrinsèque indépendante des êtres } \\
\text { humains. Ils sont posés sur un pied } \\
\text { d'égalité (D3). } \\
\text { Préserver les écosystèmes pour les } \\
\text { êtres vivants qui y habitent. } \\
\text { Responsabilité envers tous les êtres } \\
\text { vivants (D4). }\end{array}$ & $\begin{array}{l}\text { Valeur intrinsèque des êtres vivants, } \\
\text { mais aussi des écosystèmes (D5). } \\
\text { Responsabilité envers tous les êtres } \\
\text { vivants et, en particulier, envers } \\
\text { ceux qui nous sont socialement } \\
\text { proches (D6). }\end{array}$ \\
\hline 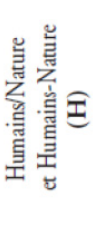 & $\begin{array}{l}\text { Les humains sont dissociés de la } \\
\text { nature (les humains ne sont pas } \\
\text { présentés comme étant des } \\
\text { animaux). Ils sont posés à } \\
\text { l'extérieur, en position } \\
\text { d'observateur, de contrôle, de } \\
\text { domination (H1). }\end{array}$ & $\begin{array}{l}\text { Les humains font partie de la } \\
\text { nature. Ils sont posés sur le même } \\
\text { pied d'égalité que les autres êtres } \\
\text { vivants (les humains sont présentés } \\
\text { comme faisant partie des animaux) } \\
\text { (H2). }\end{array}$ & $\begin{array}{l}\text { Les humains font partie de la } \\
\text { nature (humains présentés comme } \\
\text { étant des animaux) (H3). Il } \\
\text { existerait des liens } \\
\text { d'interdépendance entre les } \\
\text { dimensions naturelle et sociale } \\
\text { (H4). }\end{array}$ \\
\hline 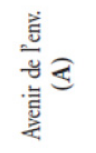 & $\begin{array}{l}\text { Ne parle que des conséquences sur } \\
\text { les humains ou sur les autres êtres } \\
\text { vivants si cela vient affecter les } \\
\text { humains (A1). }\end{array}$ & $\begin{array}{l}\text { Parle également des conséquences } \\
\text { sur les autres êtres vivants (sur les } \\
\text { animaux et les végétaux) (A2). }\end{array}$ & $\begin{array}{l}\text { Parle des conséquences sur les } \\
\text { humains et les autres êtres vivants, } \\
\text { mais aussi considère l'ensemble des } \\
\text { écosystèmes. (A3). }\end{array}$ \\
\hline
\end{tabular}

8 Les encadrés qui suivent, présentent et analysent les éléments des trois récits :

9 Récit d'Arthur (perspective anthropocentrique) 
Arthur plante un arbre dans la forêt.

Car il pense aux enfants qu'il aura plus tard et aux enfants que ceux-ci auront.

Il veut qu'ils puissent profiter des bienfaits de la forêt, qu'ils puissent admirer sa beauté, qu'ils puissent s'y amuser, s'y promener.

Les arbres sont importants, car leur bois est très utile aux humains : il permet de construire toutes sortes de choses, de faire du papier.

Les arbres sont aussi essentiels à la survie des humains. Sans les arbres, les humains ne pourraient plus respirer.

Les humains sont bien supérieurs aux animaux. Ils ont donc le pouvoir de contrôler la nature pour la protéger. Arthur est donc très fier de planter cet arbre.

D2. Responsabilité envers les générations futures

C2. Protection pour des raisons esthétiques et récréatives

C1. Environnement $=$ ressource

A1. Survie humaine

D1, H1. Humains à

l'extérieur de la nature en position de contrôle

Récit de Bastien (perspective biocentrique)

Bastien plante un arbre dans la forêt.

Car il pense aux animaux de la forêt. Il veut que les oiseaux puissent y faire leurs nids, que les écureuils puissent y vivre.

Les arbres sont importants, car ce sont des êtres vivants, eux aussi, comme les êtres humains.

Les arbres sont, aussi, essentiels à la survie de tous les êtres vivants.

Sans les arbres, les animaux, dont les humains, ne pourraient plus respirer. Les humains sont des êtres vivants, au même titre que les autres animaux et les végétaux.

Ils ont donc le devoir de préserver la vie des autres êtres vivants. Bastien est donc très fier de planter cet arbre.

D4. Responsabilite envers les autres êtres vivants

C3, D3, H2.

Certaines égalités entre les êtres vivants

A2. Conséquences pour les autres êtres vivants

C3, D3, H2.

Certaines égalités entre les êtres vivants

D4. Responsabilité envers les animaux

Récit d'Éric (perspective écocentrique) 


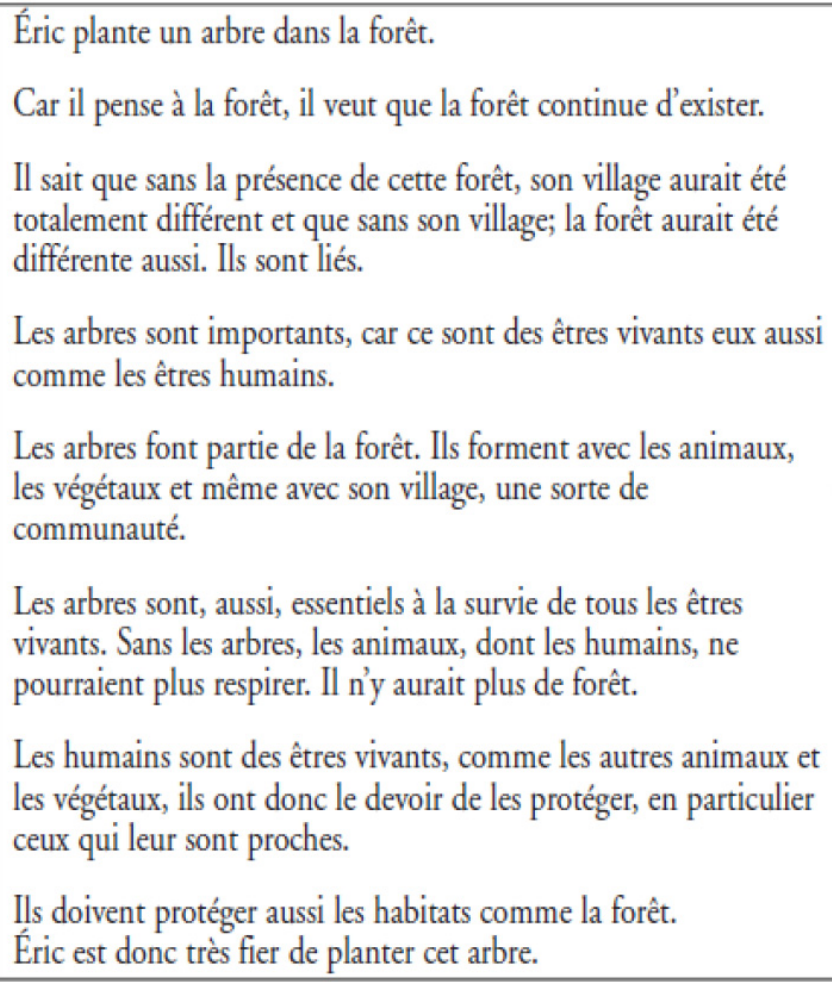

Les humains sont des êtres vivants, comme les autres animaux et les végétaux, ils ont donc le devoir de les protéger, en particulier ceux qui leur sont proches.

Ils doivent protéger aussi les habitats comme la forêt.

Éric est donc très fier de planter cet arbre.

\begin{tabular}{|l|}
\hline $\begin{array}{l}\text { D5. Prise en compte } \\
\text { de la forêt en tant que } \\
\text { telle }\end{array}$ \\
\hline $\begin{array}{l}\text { C5, H4. Interrelation } \\
\text { des dimensions } \\
\text { sociales et naturelles }\end{array}$ \\
\hline $\begin{array}{l}\text { H3. Humains font } \\
\text { partie de la nature }\end{array}$ \\
\hline $\begin{array}{l}\text { C4. Environnement } \\
\text { vu comme un éco- } \\
\text { socio-système }\end{array}$ \\
\hline $\begin{array}{l}\text { A3. Conséquences } \\
\text { pour les êtres vivants } \\
\text { et la forêt }\end{array}$ \\
\hline $\begin{array}{l}\text { D6. Responsabilité } \\
\text { envers les autres êtres } \\
\text { vivants, surtout ceux } \\
\text { qui sont socialement } \\
\text { proches }\end{array}$ \\
\hline $\begin{array}{l}\text { A3. Protection des } \\
\text { écosystèmes }\end{array}$ \\
\hline
\end{tabular}

Activité pédagogique proposée

10 Cette activité est la dernière proposée dans une séquence de plusieurs (Rousseau, 2011), mais elle peut être utilisée indépendamment des autres. Une fois que les élèves ont lu chacun des récits, ils écrivent sur une feuille les éléments, pour chacune des histoires, qui correspondent et qui ne correspondent pas, selon eux, à leur propre vision du monde. Puis, ils indiquent quel récit s'approche le mieux de leur pensée.

Chaque élève présente ensuite son choix à ses coéquipiers (équipes de 4). Ceux-ci doivent écouter la présentation de chacun des membres sans émettre de jugement, mais ils peuvent poser des questions. En effet, il s'agit ici de leur faire prendre conscience que d'autres personnes peuvent avoir des visions différentes de la leur quand on pose un geste environnemental.

L'enseignante ou l'enseignant peut ensuite lancer un débat : Les récits d'Arthur, de Bastien et d'Éric nous montrent qu'une même situation (c'est-à-dire planter un arbre) peut être vue, au moins, selon 3 points de vue différents. Cela signifie-t-il que des personnes ayant des points de vue différents pourraient avoir de la difficulté à travailler ensemble sur un même projet environnemental ? Pourquoi? Que devront-elles faire pour mieux collaborer? L'enseignante ou l'enseignant pourrait utiliser l'exemple d'un projet concret en environnement proche des élèves pour alimenter leurs propos. Il s'agit encore ici de les amener à chercher à mieux comprendre le point de vue de différents acteurs face à une même problématique socio-environnementale.

\section{Éléments de discussion}

13 Nous pensons que ces questions supportées par ces récits pourraient permettre de mieux comprendre que différents acteurs ayant des identités éthiques différentes peuvent s'engager ensemble autour d'un même acte environnemental. Ils peuvent 
également inciter les élèves à mieux argumenter leur propre position éthique. Il reste certes à expérimenter cette activité. Également, il serait intéressant de concevoir des récits adoptant d'autres perspectives éthiques que les trois que nous avons considérées.

\section{BIBLIOGRAPHIE}

Afeissa, H.-S., Dir., (2007). Éthique de l'environnement : Nature, valeur, respect. Paris : Librairie Philosophique J. Vrin. Collection : Textes Clés.

Callicott, B. (1999). Beyond the Land Ethic : More Essays in Environmental Philosophy. State University of New York Press, Albany.

Goffin, L. (1998). L'environnement comme éco-socio-système. In Loriaux, M., Populations et développement : une approche globale et systémique. Louvain-la-Neuve/Paris : Academia Bruylant/L'Harmattan, 199-230.

Larrère, C. (1997). Les philosophies de l'environnement. Paris : Presses Universitaires de France. Larrère, C. et Larrère, R. (1997). Du bon usage de la nature. Pour une philosophie de l'environnement. Paris : Éditions Aubier.

Lundmark, C. (2007). The New Ecological Paradigm Revisited : Anchoring the NEP Scale in Environmental Ethics. Environmental Education Research, 13(3), 329-347. London : Routledge.

Manoli, C., Johnson, B. et R. Dunlap. (2007). Assessing Children's Environmental Worldviews : Modifying and Validating the New Ecological Paradigm Scale for Use With Children. The Journal of Environmental Education, 38(4), 3-13. London : Routledge.

Rousseau, C. (2011), Étude des perspectives éthiques sur l'environnement : activités de dialogues pour les élèves de la fin du primaire. Québec : Mémoire de l'université Laval.

Rousseau, C. (2013). Prise en compte de la dimension éthique chez les enfants pour élaborer un référentiel en éducation aux valeurs environnementales. Éducation relative à l'environnement : regards, recherches, réflexion, 11, 283-291. Montréal : Université du Québec à Montréal.

Sauvé, L., Villemagne, C. et Orellana, I. (2003). Éléments d'une pédagogie de l'éducation relative à l'environnement. Module 4. Programme d'études supérieures - Formation en éducation relative à l'environnement - Francophonie internationale. Montréal : Les Publications ERE-UQAM, Université du Québec à Montréal - Collectif ERE-Francophonie.

\section{NOTES}

1. Les thèmes résultent de la lecture de 27 textes d'auteurs du domaine de l'éducation relative à l'environnement qui se sont intéressés aux perspectives éthiques anthropocentrique, biocentrique et écocentrique, ou qui ont traité des conceptions de la nature et l'environnement chez des populations d'adultes et d'élèves. Des ouvrages en philosophie (ou éthique) de l'environnement ont aussi été consultés (comme Larrère, 1997; Larrère et Larrère, 1997; Afeissa, 2007). Ces thèmes sont issus également de l'analyse critique approfondie du NEP Scale for Children de Manoli, Johnson et Dunlap (2007). 
INDEX

Mots-clés : activité pédagogique, anthropocentrisme, biocentrisme, écocentrisme, éthique

Keywords : pedagogical activity, anthropocentrism, biocentrism, ecocentrism, ethics

\section{AUTEUR}

\section{CARINE ROUSSEAU}

Doctorante en technologie éducative et chargée de cours à l'Université Laval. Elle a complété une maîtrise en éducation relative à l'environnement à l'Université Laval. 